\title{
SAGE III aerosol extinction validation in the Arctic winter: comparisons with SAGE II and POAM III
}

\author{
L. W. Thomason ${ }^{1}$, L. R. Poole ${ }^{2, *}$, and C. E. Randall ${ }^{3}$ \\ ${ }^{1}$ NASA Langley Research Center, Hampton, Virginia, USA \\ ${ }^{2}$ Science Applications International Corporation, Hampton, Virginia, USA \\ ${ }^{3}$ University of Colorado, Boulder, Colorado, USA \\ *now at: Science Systems and Applications, Inc., Hampton, Virginia, USA
}

Received: 21 September 2006 - Published in Atmos. Chem. Phys. Discuss.: 16 November 2006

Revised: 13 February 2007 - Accepted: 28 February 2007 - Published: 8 March 2007

\begin{abstract}
The use of SAGE III multiwavelength aerosol extinction coefficient measurements to infer PSC type is contingent on the robustness of both the extinction magnitude and its spectral variation. Past validation with SAGE II and other similar measurements has shown that the SAGE III extinction coefficient measurements are reliable though the comparisons have been greatly weighted toward measurements made at mid-latitudes. Some aerosol comparisons made in the Arctic winter as a part of SOLVE II suggested that SAGE III values, particularly at longer wavelengths, are too small with the implication that both the magnitude and the wavelength dependence are not reliable. Comparisons with POAM III have also suggested a similar discrepancy. Herein, we use SAGE II data as a common standard for comparison of SAGE III and POAM III measurements in the Arctic winters of 2002/2003 through 2004/2005. During the winter, SAGE II measurements are made infrequently at the same latitudes as these instruments. We have mitigated this problem through the use of potential vorticity as a spatial coordinate and thus greatly increased of the number of coincident events. We find that SAGE II and III extinction coefficient measurements show a high degree of compatibility at both $1020 \mathrm{~nm}$ and $450 \mathrm{~nm}$ except a 10-20\% bias at both wavelengths. In addition, the 452 to $1020 \mathrm{~nm}$ extinction ratio shows a consistent bias of $\sim 30 \%$ throughout the lower stratosphere. We also find that SAGE II and POAM III are on average consistent though the comparisons show a much higher variability and larger bias than SAGE II/III comparisons. In addition, we find that SAGE II and POAM III data sets are not well correlated at and below $18 \mathrm{~km}$. Overall, we find both the extinction values and the spectral dependence from SAGE III are robust and we find no evidence of a significant defect within the Arctic vortex.
\end{abstract}

Correspondence to: L. W. Thomason

(1.w.thomason@larc.nasa.gov)

\section{Introduction}

The Stratospheric Aerosol and Gas Experiment III (SAGE III) produced profiles from the mid-troposphere to the mesosphere of ozone, $\mathrm{NO}_{2}$, water vapor, and multiwavelength aerosol extinction between February 2002 and March 2006. Due to orbital considerations, these profiles were made primarily between 50 and $80^{\circ} \mathrm{N}$ and 25 and $60^{\circ} \mathrm{S}$. During Arctic winter SAGE III sunset observations occurred at latitudes greater than $60^{\circ} \mathrm{N}$ and produced numerous profiles within the Arctic vortex including frequent observations of polar stratospheric clouds (PSCs). Since SAGE III made aerosol extinction measurements from $385 \mathrm{~nm}$ to $1545 \mathrm{~nm}$, these measurements have the potential to allow the inference of PSC microphysical properties (Poole et al., 2003). Single-wavelength $(\sim 1 \mu \mathrm{m})$ aerosol extinction data from SAM (Stratospheric Aerosol Measurement) II, POAM (Polar Ozone and Aerosol Measurement) II/III, and SAGE II have provided much of our present knowledge of PSC climatology (McCormick et al., 1982; Poole and Pitts, 1994; Fromm et al., 2003). In these studies, PSCs were identified as those measurements having 1- $\mu \mathrm{m}$ extinction coefficients significantly larger than the background (non-PSC) aerosol extinction. This approach provides reasonably accurate statistics on PSC occurrence, but it obviously excludes any PSCs with extinctions below the detection threshold, and it provides little information on PSC particle properties. Several recent studies have shown that a dual-wavelength analysis of extinction data provides significantly enhanced information on PSC microphysics, in particular the ability to discriminate liquid and solid particles. For example, Strawa et al. (2002) showed that multiwavelength POAM III aerosol extinction data is consistent with the observation of supercooled ternary solution (STS) and nitric acid trihydrate (NAT) PSC particles. Poole et al. (2003) found similar results using 2 SAGE III channels and also found evidence for mixtures of STS with very few relatively large NAT particles (so-called "NAT

Published by Copernicus GmbH on behalf of the European Geosciences Union. 
Table 1. Aerosol extinction coefficient wavelengths for SAGE II, SAGE III, and POAM III in nanometers.

\begin{tabular}{lll}
\hline SAGE II & SAGE III & POAM III \\
\hline 386 & 385 & 354 \\
452 & 449 & 442 \\
525 & 521 & 600 \\
1020 & 600 & 779 \\
& 676 & 920 \\
& 755 & 1018 \\
& 868 & \\
& 1020 & \\
& 1545 & \\
\hline
\end{tabular}

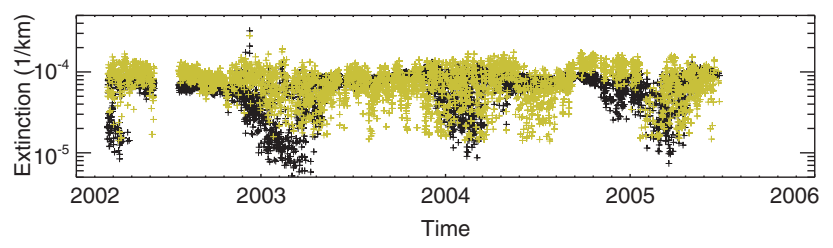

Fig. 1. This figure shows the $18-\mathrm{km}, 1020-\mathrm{nm}$ aerosol extinction coefficient measured by SAGE III (black) and POAM III (gold) in the Northern Hemisphere. Note that this figure shows all observations by each instrument with no matching requirement.

rocks") based on the multiwavelength analysis.

The use of SAGE III multispectral aerosol extinction data to infer PSC composition is plainly dependent on the quality of the extinction measurements including the spectral variation. While the works cited above indicate that these data are useful in this application, there is some question regarding the overall quality of the SAGE III aerosol data. Russell et al. (2004) made measurements of the multispectral dependence of aerosol column optical depth above NASA DC-8 flight altitudes $(\sim 12 \mathrm{~km})$ as a part of the SAGE III Ozone Loss and Validation Experiment (SOLVE II) using the Ames Airborne Tracking Sunphotometer (AATS-14). They found that AATS-14 optical depths were generally larger than the integrated column SAGE III values, particularly at longer wavelengths where the discrepancy could reach a factor of 3. Similarly, Russell et al. (2004) note that, while SAGE III and measurements made by POAM III are consistent outside of the polar vortex, SAGE III values are consistently lower within the Arctic vortex. This is demonstrated in Fig. 1 which shows a time series of northern hemisphere SAGE III and POAM III 1020-nm aerosol extinction measurements at $18 \mathrm{~km}$. SAGE III observes many more low aerosol extinction coefficient values $\left(<2 . \times 10^{5} \mathrm{~km}^{-1}\right)$ during winter months (particularly in the winter of 2002/2003) than does POAM III while conversely showing much less variability during the summer months. Nominally, smaller aerosol ex- tinction coefficients within the polar vortices compared to mid-latitude values is a well known phenomenon (Kent el al., 1985; Curtius et al., 2005; Thomason and Poole, 1993) that has been taken into account in PSC identification schemes (e.g., Poole and Pitts, 1994).

To facilitate the use of SAGE III measurements in PSC composition studies and illuminate the differences between SAGE III and POAM III we have conducted an extensive comparison of multiwavelength extinction measurements by SAGE III and POAM III with observations made by SAGE II (1984-2005). While SAGE III and SAGE II are more similar to each other than to POAM III, they also have significant differences in hardware configuration and operation. In addition, the SAGE III and SAGE II algorithms that produce the data products including aerosol extinction coefficient, while following similar approaches, are also distinct and have minimal common software. Thus, we do not believe that there is any inherent predilection for the two SAGE instruments to agree at the expense of POAM III and that comparison of these three instruments will provide insight into the source of reported disagreements. Matching SAGE II events to SAGE III and POAM III is a non-trivial task since SAGE II is in an inclined orbit and, as a result, makes relatively few observations at high latitudes particularly in winter. However, by combining such coincidences over the three winters in which data from all 3 instruments are available (2002/2003 through 2004/2005) and using potential vorticity as a spatial coordinate rather than latitude, we find roughly 200 coincidences for SAGE III and SAGE II and roughly 100 coincidences for POAM III and SAGE II. Herein, we will describe the three instruments and highlight where they and their processing algorithms are different. We then show the results of the data intercomparisons and discuss the results.

\section{Instrument and algorithm descriptions}

All three instruments are solar occultation devices and share the fundamental strengths and weaknesses of this approach. The instruments observe the change in the apparent brightness of the Sun as it is obscured (or "occulted") by the Earth's atmosphere during each sunrise and sunset encountered by the spacecraft or about 30 times per day. A line-of-sight (LOS) transmission is computed by dividing the observed through-the-atmosphere intensity by a value measured above the atmosphere. By measuring the LOS transmission at multiple wavelengths it is possible to produce profiles of gas species such as ozone, $\mathrm{NO}_{2}$, and water vapor as well as the spectral dependence of aerosol extinction coefficient. The occultation method is well suited to stratospheric applications where the optical depths of these species are low and where the horizontal variability is small since the horizontal scale of the measurements is on the order of hundreds of kilometers and sampling is sparse (Thomason et al., 2003). SAGE III uses 87 channels between 290 and $1545 \mathrm{~nm}$ to 
produce profiles of ozone, $\mathrm{NO}_{2}$, water vapor, temperature, and aerosol extinction coefficient at the 9 wavelengths shown in Table 1 (SAGE III ATBD, 2002). POAM III uses 9 channels between 354 and $1018 \mathrm{~nm}$ to produce profiles of ozone, $\mathrm{NO}_{2}$, and water vapor as well as aerosol extinction at the 6 wavelengths shown in Table 1 (Lumpe et al., 2002). SAGE II is the least complex of these instruments with only 7 channels from which ozone, $\mathrm{NO}_{2}$, water vapor and aerosol extinction coefficient at 4 wavelengths are produced (Chu et al., 1989; Thomason et al., 2000). We will limit our discussion to the current releases for all instruments: SAGE III (Version 3), POAM III (Version 4), and SAGE II (Version 6.2). In addition, since all three instruments make aerosol extinction coefficient measurements near $1020 \mathrm{~nm}$ and $450 \mathrm{~nm}$, we will focus on these common measurements.

In addition to the differences in the number of channels, there are other differences in how the instruments operate. For instance, SAGE II and SAGE III instruments use a mirror to scan across the Sun normal to the horizon continuously during an event. The spacecraft ephemeris and the times of crossing the Sun edges form the basis for determining the altitude of the on-Sun measurements. In the lower atmosphere, where the lower edge of the Sun may be totally obscured, the time of crossing the upper Sun edge and the measured mirror scan rate is used for altitude registration. On the other hand, POAM III stares at the center of brightness of the Sun. With no atmospheric attenuation, the physical center and the center of brightness are the same. However, for Sun positions below $\sim 40 \mathrm{~km}$, the center of brightness moves to a higher position on the Sun due to the effects of refraction and the opacity of the atmosphere. At altitudes above $\sim 26 \mathrm{~km}$, the pointing position is determined from the optical depth ratios of Rayleigh dominated channels. Below $\sim 26 \mathrm{~km}$, pointing is determined from changes in the elevation angle of the optical head as recorded by the instrument. Thus all three instruments are dependent on knowledge of a Sun positiondependent exoatmospheric Sun brightness curve to produce transmission profiles from the measured signals. The field of view of the instruments are 0.5 by 1.5 arc minutes for SAGE III, 0.5 by 5 arc minutes for SAGE II, and 0.8 by 48 arc minutes for POAM III (the unrefracted Sun is 30 arc minutes wide) where the first number denotes the vertical field of view and is a key parameter in defining the vertical resolution. POAM III has a slightly coarser vertical resolution than the SAGE instruments but this should not be relevant to the following discussions.

SAGE III and SAGE II use a similar approach for the conversion of the measured LOS transmission profiles to LOS product profiles. The effects of molecular scattering are computed using temperature and pressure profiles obtained from the National Center for Environmental Prediction (NCEP). The remaining optical depth $(-\log ($ transmission$))$ is separated into contributions by gas species and aerosol using a least squares approach (Chu et al., 1989; Thomason et al., 2000). For SAGE II, the contributions of aerosol in nominal gas channels at $448\left(\mathrm{NO}_{2}\right)$ and $600 \mathrm{~nm}$ (ozone) are approximated as linear combinations of the aerosol LOS optical depth at 452,525 , and $1020 \mathrm{~nm}$ where the coefficients for these relationships have been derived based on the relationship predicted by single mode log-normal size distributions for sulfate aerosol at stratospheric temperatures (Thomason et al., 2001). The aerosol contribution at $386 \mathrm{~nm}$ and in the water vapor channel are computed after the primary processing. SAGE III uses a multi-linear regression (MLR) technique with the twenty 1 -nm wide $\mathrm{NO}_{2}$ channels between 430 and $450 \mathrm{~nm}$ and the ten $7-\mathrm{nm}$ wide ozone channels between 560 and $630 \mathrm{~nm}$. The LOS aerosol contribution is produced as a residual following the subtraction of the contributions from other components. These include the a priori molecular density profile (from NCEP) and the retrieved ozone and $\mathrm{NO}_{2}$ values as well as the contribution of water vapor (where appropriate) that is retrieved using a non-linear least squares method prior to the application of the MLR technique. Since there is essentially no contribution by ozone at $1020 \mathrm{~nm}$, this channel for both SAGE III and SAGE II is only dependent on the measured transmission and the a priori molecular density profile.

POAM III uses an optimal estimation technique that simultaneously solves for the target gas species and the coefficients to an empirical function that accounts for the effects of aerosol. In this model, aerosol LOS optical depth, $\delta_{i}$, is given by

$\delta_{i}=\mu_{o}+\mu_{1} \kappa_{i}+\mu_{2} \kappa_{i}^{2}$,

where the $\mu_{j}$ are the effective aerosol coefficients retrieved in the algorithm, and $\kappa_{i} \equiv \ln \left(\lambda_{i}\right)$, with $\lambda_{i}$ being the central wavelength of channel $i$. This relationship has been found to be an adequate representation of the spectral dependence predicted by stratospheric aerosol models (Lumpe et al., 2002). In describing the previous version (Version 3), Lumpe et al. (2002) found that the aerosol extinction coefficient at $442 \mathrm{~nm}$ is not highly coupled to the extinction at other wavelengths while the value at $1020 \mathrm{~nm}$ is significantly coupled to values measured at 779 and $920 \mathrm{~nm}$ at all altitudes and to shorter wavelengths above $20 \mathrm{~km}$. For all three instruments, species are separated using LOS values, and those profiles are subsequently "peeled" to the vertical data product profiles.

Randall et al. (2001) compared version 3.0 POAM III aerosol extinction to version 6.0 SAGE II data. They showed that at $1 \mu \mathrm{m}$ the instruments agreed to within $\pm 30 \%$ from $10-22 \mathrm{~km}$, but that there was significantly more variability in the POAM measurements. Both the SAGE II and POAM III algorithms have been improved since then; analogous comparisons with the newer versions show agreement to within $\pm 10 \%$ at $1 \mu \mathrm{m}$ from $15-24 \mathrm{~km}$, with larger disagreements $( \pm 30 \%)$ down to $11 \mathrm{~km}$. Substantial variability in the POAM data remains, however, even in the version 4.0 POAM retrievals. The precision at $450 \mathrm{~nm}$ was similar between the two instruments, but systematic biases existed that were attributed to problems with the v6.0 SAGE II retrieval 

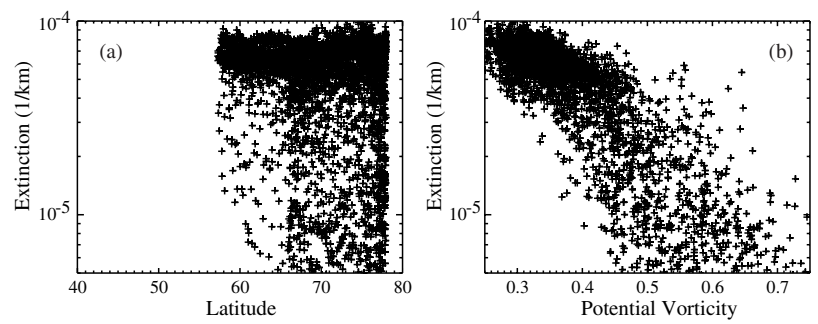

Fig. 2. This figure depicts a comparison of the distribution of 1020$\mathrm{nm}$ aerosol extinction coefficient from SAGE III in January through March of 2003 through 2005 at $18 \mathrm{~km}$ in the northern hemisphere. Frame (a) shows the distribution as a function of latitude while frame (b) shows the distribution as a function of potential vorticity in units of $\mathrm{km}^{2} \mathrm{~s}^{-1} \mathrm{~kg}^{-1} \times 10^{4}$.
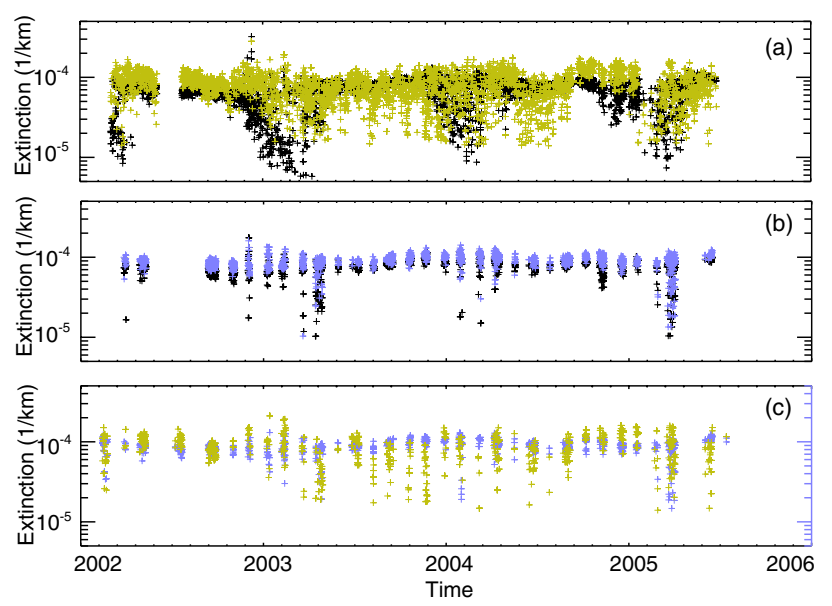

Fig. 3. This figure shows $18 \mathrm{~km}, 1020-\mathrm{nm}$ aerosol extinction coefficient measured by SAGE III, SAGE II, and POAM II using the match requirements described in the text. Frame (a) shows SAGE III (black) matched with POAM III (gold); frame (b) shows SAGE III (black) matched with SAGE II (blue); and frame (c) shows POAM III (gold) matched with SAGE II (blue).

algorithm. These problems have been fixed in the version 6.2 algorithm used here, and comparisons of POAM III data in the northern hemisphere with the newer retrievals agree to within $\pm 20 \%$ from $13-21 \mathrm{~km}$, increasing to $\pm 30-40 \%$ above and below this range.

\section{Comparisons}

Figure 2 shows the distribution of SAGE III 1020-nm extinction coefficient measurements at $18 \mathrm{~km}$ north of $45^{\circ} \mathrm{N}$ for the winters of 2002/2003 to 2004/2005. In frame (a), the data is plotted as a function of latitude and in frame (b) it is plotted as a function of Ertel potential vorticity (PV) expressed in units of $\mathrm{km}^{2} \mathrm{~s}^{-1} \mathrm{~kg}^{-1} \times 10^{4}$. SAGE III and SAGE II auxiliary data sets which contain PV, equivalent latitude and other dynamical information have been produced by Gloria Manney (Manney et al., 2001) using National Centers for Environmental Prediction (NCEP) analyses and are available at $\mathrm{ftp} / / / \mathrm{mls}$.jpl.nasa.gov/pub/outgoing/manney. PV is a dynamical tracer and as such more useful than latitude for matching event locations in the Arctic winter since it can better characterize the air mass in which the measurements are made particularly in the presence of strong PV and species gradients across the edge of the wintertime polar vortex. It is clear that the use of PV greatly increases the organization of the distribution and illustrates the strength of using this coordinate particularly in the vicinity of the Arctic vortex. As a result, we use time, longitude, altitude, and PV as coordinates for determining coincidences. Since, as previously noted, there are relatively few opportunities to match SAGE II events in the wintertime Arctic, we found that we needed to use relatively broad match criteria to maximize the number of coincident events. Thus the noise in the matches is likely larger than would be observed with tighter match criteria due to actual geophysical variability. We used identical altitudes, \pm 1 day, $\pm 24^{\circ}$ longitude, and $\pm 5 \%$ of the PV range observed in the SAGE II data for observations in northern hemisphere winters. This latter value is very roughly the equivalent of a $2^{\circ}$ range in latitude. Since, as will be shown below, all three instruments' observations have roughly the same range in potential vorticity, the opportunity exists to match events for the entire domain of PV. We find some coincidences by these criteria where the latitude difference approaches $10^{\circ}$. Including these points increased the standard deviation of the comparisons but had little impact on the mean values. As a compromise between increasing the number of coincidences by opening the coincidence window and decreasing variance by restricting spatial differences, we also include a limit of $5^{\circ}$ difference in latitude. We also eliminated all coincidences where relative errors are greater than $75 \%$ and where $1020-\mathrm{nm}$ extinction values are greater than $4 \times 10^{-4} \mathrm{~km}^{-1}$ and temperatures are less than $200 \mathrm{~K}$ as a first cut at removing PSCs. We choose to eliminate most PSCs since they tend to be spatially inhomogeneous and, for the geometry of these instruments, matching PSC events yield extremely different results simply due to spatial variability. It is not critical to eliminate all PSCs but restricting comparisons to low values of extinction mitigates the inhomogeneity problem. This is mostly accomplished through the use of the temperature cutoff where the extinction cutoff removes a few large values that escape the temperature filter. This complicates the comparison however the biggest outstanding issue for SAGE III extinction data quality is at low values. In addition, solar occulation measurements of aerosol extinction improve in quality for increasing magnitudes of extinction so it is not unreasonable to expect the results for low extinctions to persist into the higher values exhibited by PSCs. The value used for the relative error limit made virtually no difference in the quality and quantity of matches except above $22 \mathrm{~km}$ where significant numbers of POAM III 


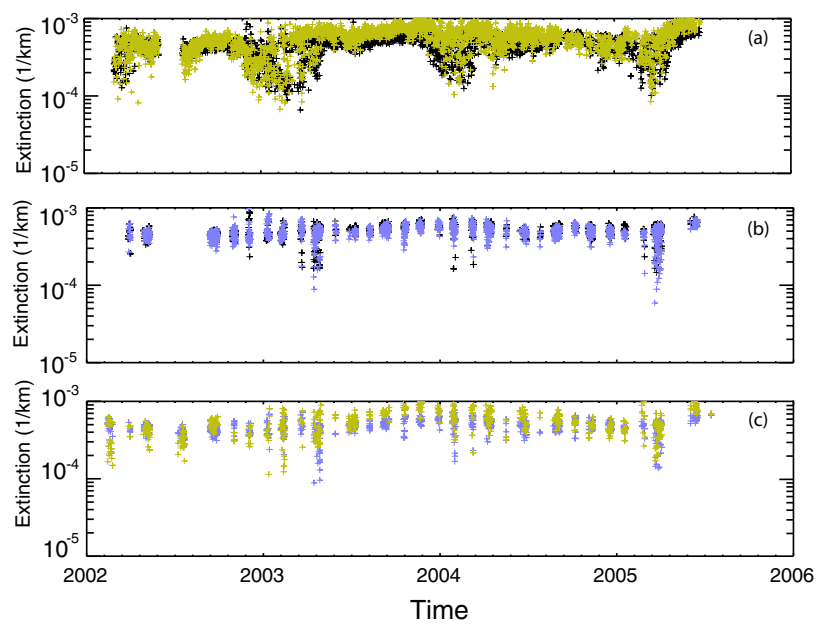

Fig. 4. This figure shows $18 \mathrm{~km}$, aerosol extinction coefficient near 450-nm as measured by SAGE III, SAGE II, and POAM II using the match requirements described in the text. Frame (a) shows POAM III (gold) matched with SAGE III (black); frame (b) shows SAGE III (black) matched with SAGE II (blue); and frame (c) shows SAGE II (blue) matched with POAM III (gold).

events are eliminated by a criterion more restrictive than that required by the SAGE instruments. As reported by Lumpe et al. (2002) POAM III extinction measurements report significantly larger uncertainties, particularly at $1020 \mathrm{~nm}$, than those associated with either SAGE data set.

Figure 3 shows the match time history for matching SAGE III and POAM III (a), SAGE III and SAGE II (b), and POAM III and SAGE II (c) coincidences at $18 \mathrm{~km}$ using the above match criteria. Clearly Fig. $3 \mathrm{a}$ is very similar to Fig. 1 and reflects the similarity in sampling that POAM III and SAGE III have in the Northern Hemisphere. At the same time, the differences between SAGE III and POAM III are unchanged by the more robust matching criteria reinforcing the idea that the differences are in fact real differences. On the other hand, the matched SAGE III and SAGE II events, as shown in Fig. 3b, demonstrate a strong qualitative agreement with fewer though still substantial number of matches. Both show a tight clustering during most of the year with some scatter toward lower values in the winter. In Fig. 3c, the matched POAM III and SAGE II events appear similar to the POAM III-SAGE III comparisons shown in Fig. 3a. Here, the POAM III data show greater variability than the SAGE II at all times particularly in the summer of 2003. This is consistent with the POAM III validation results of Randall et al. (2001). The late winter POAM III data also show a similar scatter toward lower values as SAGE II that may be consistent with that observed between SAGE III and SAGE II. Figure 4 shows the same analysis except using the measurement channels located near $450 \mathrm{~nm}$. For this set of measurements, the variability of the three data sets is far more consistent than is found for the 1020-nm comparisons. There
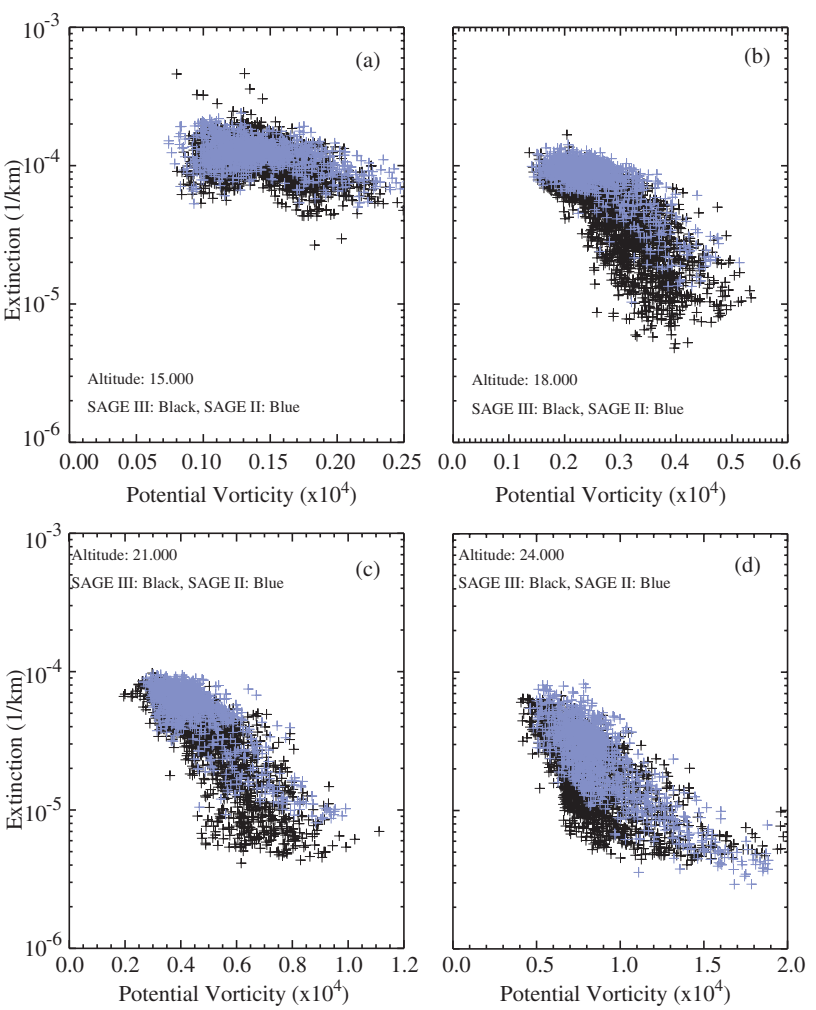

Fig. 5. This figure shows the distribution of 1020-nm aerosol extinction coefficient measured by SAGE III (black) and SAGE II (blue) at 15 (a), 18 (b), 21 (c), and $24 \mathrm{~km}$ (d) for January through March of 2003 through 2005. The plots show all data without any matching requirements.

are systematic differences between the three data sets but all show low variability during the summer and a substantial expansion of the aerosol extinction coefficient domain toward lower values during the winter months. All three data sets reach similar minimum values of 450-nm extinction during the winter periods, around $10^{-4} \mathrm{~km}^{-1}$. This is in contrast to the comparisons at $1020 \mathrm{~nm}$, where particularly during the 2002-2003 winter minimum POAM III extinction values were significantly higher than from SAGE III.

Since our goal is to verify SAGE III's suitability for PSC studies, we now take a closer look at the matches that occur during the Arctic winter (January-March; 2003-2005) at both 1020 and $450 \mathrm{~nm}$ including the extinction or "color" ratios since they play such a crucial role in PSC type determination. Figures 5 and 6 show a winter-only comparison of 1020-nm aerosol extinction coefficients as a function of PV. In Fig. 5, we show the distribution of SAGE III and SAGE II 1020-nm extinction at 15, 18, 21, and $24 \mathrm{~km}$ as a function of PV for the three focus winters. Given the differences in sampling locations, times of measurements, and the mixture of years, some variation in the ensemble of extinctions is expected. There is no matching of events in this case. Nonetheless we see a similar distribution of values 

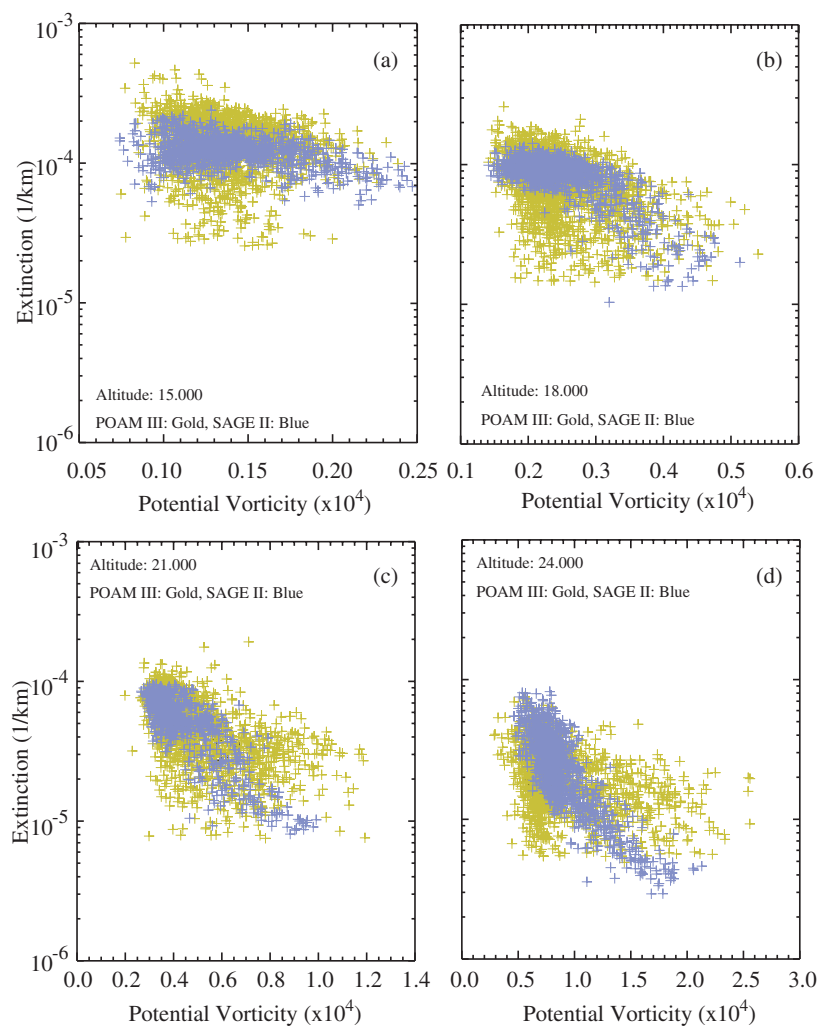

Fig. 6. This figure shows the distribution of 1020-nm aerosol extinction coefficient measured by POAM III (gold) and SAGE II (blue) at 15 (a), 18 (b), 21 (c), and $24 \mathrm{~km}$ (d) for January through March of 2003 through 2005. The plots show all data without any matching requirements.

for two instruments though SAGE II has significantly fewer measurements at high PV values than does SAGE III. Other than the $15 \mathrm{~km}$ altitude, we observe a significant negative tilt in the aerosol extinction dependence on PV. For instance, the mean value drops by nearly an order of magnitude across the domain of PV. Figure 6 shows the same comparisons for POAM III and SAGE II (the same set of points shown in Fig. 5). POAM III also shows a tilt with PV but, as would be expected from Fig. 3, with a much larger variation in extinction coefficient at all values of PV. As a result, POAM III 1020-nm extinction coefficients are more weakly correlated with PV than either SAGE II or SAGE III. Since POAM III uses a different PV source than SAGE II/III, it is possible that differences between the PV products could produce an apparently noisy outcome for POAM III. This does not seem likely to be the sole factor since the POAM III summer data still show (shown in Fig. 3) far more variation in extinction coefficient than either SAGE instrument in a period with much weaker PV gradients.

Figure 7 shows the scatter of SAGE III versus SAGE II 1020-nm extinction coefficient limited to data pairs that satisfy our match criteria. We perform our statistical averaging
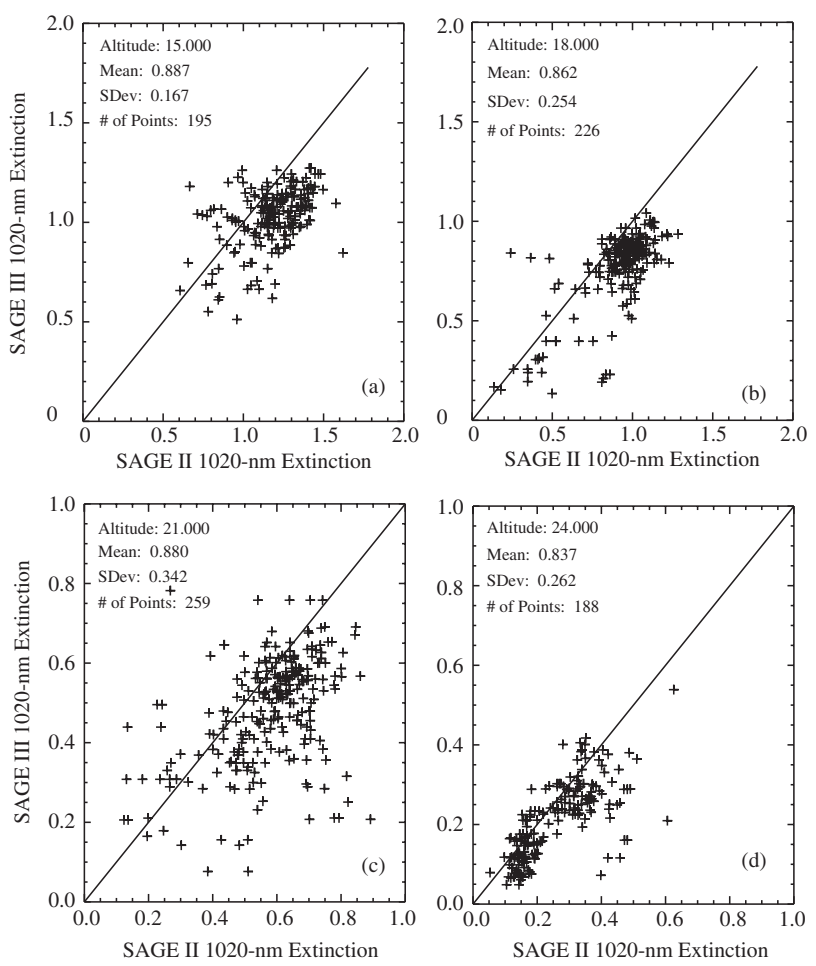

Fig. 7. This figure shows the relationship between SAGE III and SAGE II 1020-nm aerosol extinction coefficient pairs matched using the criteria described in the text for 15 (a), 18 (b), 21 (c), and $24 \mathrm{~km}$ (d). All extinctions are multiplied by $10^{4}$. The mean statistics shown were computed using the ratio defined in Eq. (2). The solid line has a slope of 1 .

on the ratio, $r$, of the data pairs as given by

$r=\frac{k_{i}(\lambda)}{k_{\mathrm{SAGEII}}(\lambda)}$

where $k_{i}(\lambda)$ is the aerosol extinction coefficient at wavelength $\lambda$ for either SAGE III or POAM III and $k_{\text {SAGEII }}(\lambda)$ is the corresponding aerosol extinction coefficient for SAGE II. We use the ratio rather than absolute values to even out the importance of the low values (that are of particular interest) with the large values that would otherwise dominate the statistical calculations. The statistics shown in Figs. 7, 8, 10, and 11 and summarized in Figs. 9 and 12 are based on this parameter. In these figures, we find that the 1020 -nm data from SAGE III and SAGE II are well correlated with a mean of $r$ between 0.83 and 0.90 or a mean difference between -17 and $-10 \%$. These values are consistent with the previously reported bias of up to $20 \%$ between SAGE III and SAGE II (Thomason and Taha, 2003). The ratio standard deviation values run between 0.15 and 0.35 for roughly 200 matches. Each of the scatter plots shows a fairly tidy primary cluster with a noisier tail toward lower extinction values. The correlation coefficient, R, (the linear Pearson correlation coefficient) between these data sets varies between 0.3 and 0.7 . 

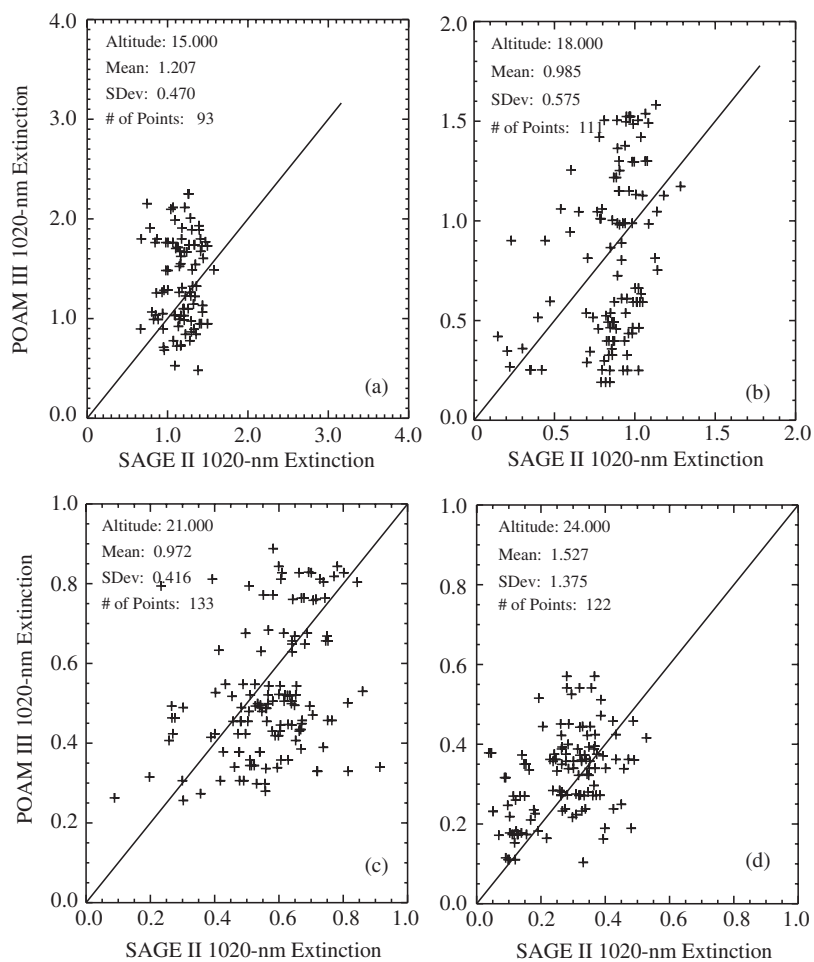

Fig. 8. This figure shows the relationship between SAGE II and POAM III 1020-nm aerosol extinction coefficient pairs matched using the criteria described in the text for 15 (a), 18 (b), 21 (c), and $24 \mathrm{~km}$ (d). All extinctions are multiplied by $10^{4}$. The mean statistics shown were computed using the ratio defined in Eq. (2). The solid line has a slope of 1 .

An increase in the noise of the matches is not unexpected due to the lower extinction values and imperfect matching of events using PV in regions with apparently high variability in extinction. Nonetheless, it is clear that the occurrence of low extinction measured by one instrument is an indicator of low extinction for the other instrument and the overall domain of extinction coefficient measured by the two instruments is very similar. There are some indications of an increased bias between the two instruments at the lowest extinctions with SAGE III more than $20 \%$ less than SAGE II values. However, the noise in the matches makes it difficult to assert this with any certainty. Figure 8 shows the scatter of the approximately 100 matches between POAM III and SAGE II. Here, the mean of the ratios varies from 0.92 to 1.5 with standard deviations of 0.38 to 1.37 or about twice that found between the SAGE instruments. The noise found in this comparison is greatly driven by noise in the POAM III $1-\mu \mathrm{m}$ data previously discussed. In this figure, while on average the agreement is fairly good, correlation between the data sets is poor particularly below $20 \mathrm{~km}$. The correlation coefficient is between 0.0 and 0.5 increasing toward higher altitudes.

Figure 9 shows a summary of the 1020-nm comparison as a function of height for the SAGE III to SAGE II compari-
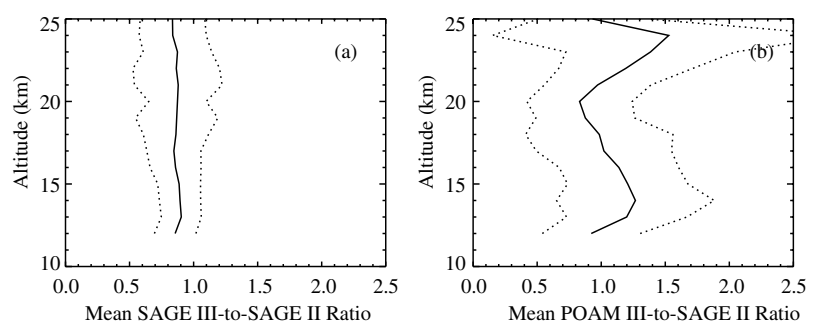

Fig. 9. (a) Mean profile of the ratio of SAGE III and SAGE II 1020$\mathrm{nm}$ aerosol extinctions coefficients for the northern hemisphere winters of 2003 through 2005 . The solid line is the mean computed using the ratio defined in equation 2 while the dotted lines are the 1 -sigma confidence limits. (b) Same as frame (a) except for POAM III and SAGE II.
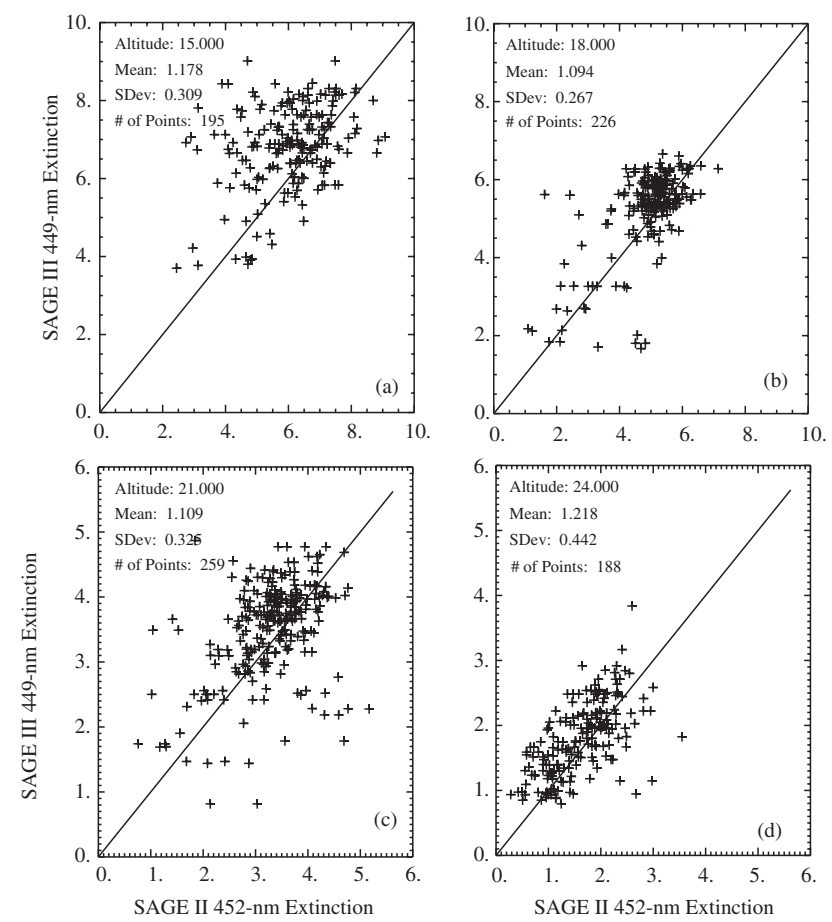

Fig. 10. This figure shows the relationship between SAGE III and SAGE II short wavelength aerosol extinction coefficient pairs (449 and $452 \mathrm{~nm}$ ) matched using the criteria described in the text for 15 (a), 18 (b), 21 (c), and $24 \mathrm{~km}$ (d). All extinctions are multiplied by $10^{4}$. The mean statistics shown were computed using the ratio defined in Eq. (2). The solid line has a slope of 1 .

son and the POAM III to SAGE II comparison. We find that the SAGE III and SAGE II comparison has a consistent bias of $\sim 15 \%$ that increases slightly with altitude. The standard deviation of the ratio also shows an increase with altitude going from $15 \%$ to near $35 \%$. The POAM III to SAGE II profile shows greater structure but averages to a similar bias (though in the opposite sense) to the SAGE III/SAGE II analysis. In this comparison, the standard deviation varies between $40 \%$ 

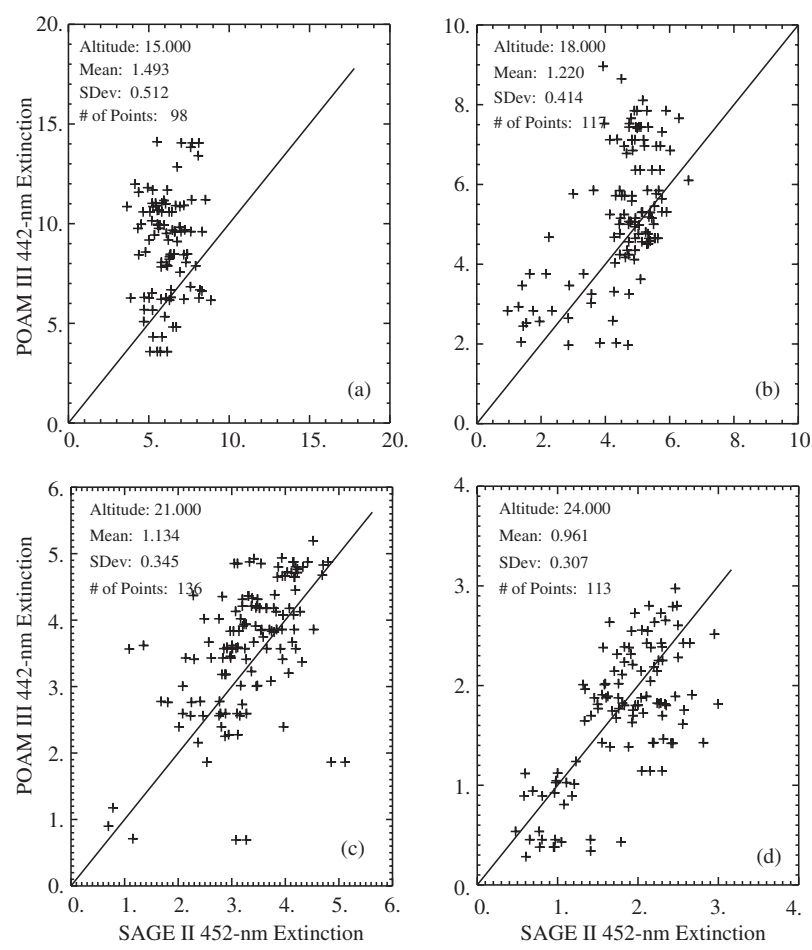

Fig. 11. This figure shows the relationship between SAGE II and POAM III short wavelength aerosol extinction coefficient pairs (452 and $442 \mathrm{~nm}$ ) matched using the criteria described in the text for 15 (a), 18 (b), 21 (c), and $24 \mathrm{~km}$ (d). All extinctions are multiplied by $10^{4}$. The mean statistics shown were computed using the ratio defined in Eq. (2). The solid line has a slope of 1.

and $140 \%$ over the depth of the profile. The good agreement with SAGE II data argues that the SAGE III 1020-nm extinction coefficient data is reliable.

Figures 10 and 11 are complementary to Figs. 7 and 8 for the comparisons at short wavelengths. Since the wavelengths are not the same for these wavelength channels, we expect some differences simply due to the wavelength dependence of aerosol extinction coefficient. For the SAGE III and SAGE II pair (449 and $452 \mathrm{~nm}$ ), this could be as large as $2 \%$ while for POAM III and SAGE II (442 and $452 \mathrm{~nm}$ ), it could be as large as $5 \%$. Just by eye it is clear that both comparisons are noisier than at $1020 \mathrm{~nm}$. This is expected since the shorter wavelengths are more strongly influenced by molecular scatter and absorption by gases. As observed for the 1020-nm comparison, the SAGE III and SAGE II comparison shows little variation with altitude. The mean ratio varies from 1.08 to 1.22 , the standard deviation is between 0.24 and 0.62 , and the correlation coefficient is between 0.24 and 0.72 , where all generally increase with altitude. On the other hand, we find that the correspondence between POAM III and SAGE II is better for this pair than at $1020 \mathrm{~nm}$. The mean has a similar range of 0.95 to 1.52 but the standard deviation is between 0.27 and 0.55 or about $2 / 3$ of that for the 1020 -nm compari-
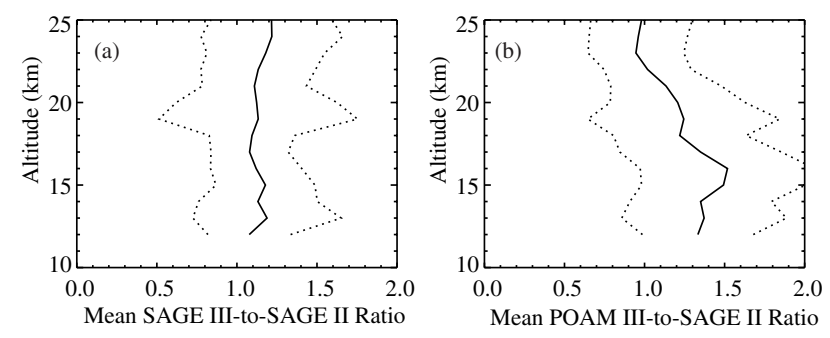

Fig. 12. (a) Mean profile of the ratio of SAGE III $449 \mathrm{~nm}$ and SAGE II 452-nm aerosol extinctions coefficients for the northern hemisphere winters of 2003 through 2005 computed using ratio defined in Eq. (2). The solid line is the mean while the dotted lines are the 1-sigma confidence limits. (b) Same as frame (a) except for POAM III $442 \mathrm{~nm}$ and SAGE II 452-nm aerosol extinction coefficient.

son and very similar to that found for the SAGE III/SAGE II short wavelength comparison. In addition, above $18 \mathrm{~km}$, the correlation coefficient is similar to or larger than the correlation between SAGE III and SAGE II measurements and is consistently greater than 0.5 . On the other hand, at and below $18 \mathrm{~km}$ the correlation coefficient between POAM II and SAGE II is close to zero. Again, even below $18 \mathrm{~km}$, we conclude that the good agreement between SAGE II and SAGE III suggests that the SAGE III 449-nm extinction coefficient data is reliable. The short wavelength summary is shown in Fig. 12. As with the 1020-nm comparison, the SAGE III and SAGE II short wavelength comparison is consistent with altitude with a bias between 10 and $20 \%$ and the standard deviation between 25 and $60 \%$. The POAM III/SAGE II comparison is significantly better behaved than the $1020-\mathrm{nm}$ comparison. While the mean is similar (ranging from 0.95 to 1.5 over the profile), the standard deviations are much less than at $1020 \mathrm{~nm}$ and vary from 27 to $55 \%$.

Since the wavelength ratio is such an important component of the ability to infer PSC composition type, we have also compared the $\sim 450$ to 1020 -nm aerosol extinction coefficient color ratios for the two instruments where the color ratio, $r$, is given by

$r=\frac{k_{i}(450 \mathrm{~nm})}{k_{i}(1020 \mathrm{~nm})} \times \frac{k_{\text {SAGEII }}(1020 \mathrm{~nm})}{k_{\text {SAGEII }}(452 \mathrm{~nm})}$,

where the " $i$ " subscript denotes either the SAGE III or POAM III instruments. Figure 13 shows the results of this analysis for both SAGE III and POAM III at $18 \mathrm{~km}$; these results are typical of what is seen between 15 and $25 \mathrm{~km}$. The results are summarized in Fig. 14. For SAGE III, the mean of the ratio of the extinction ratios is nearly constant at 1.3 below $21 \mathrm{~km}$ and increases above that altitude to near 1.5 at $25 \mathrm{~km}$. Similarly the standard deviation is nearly constant below $21 \mathrm{~km}$ at a value of 0.2 and increases above that altitude to 0.5 . While the mean value of the color ratio can be inferred from previous figures, the low standard deviation in the ratio is less obvious and arises out of the fact that the 

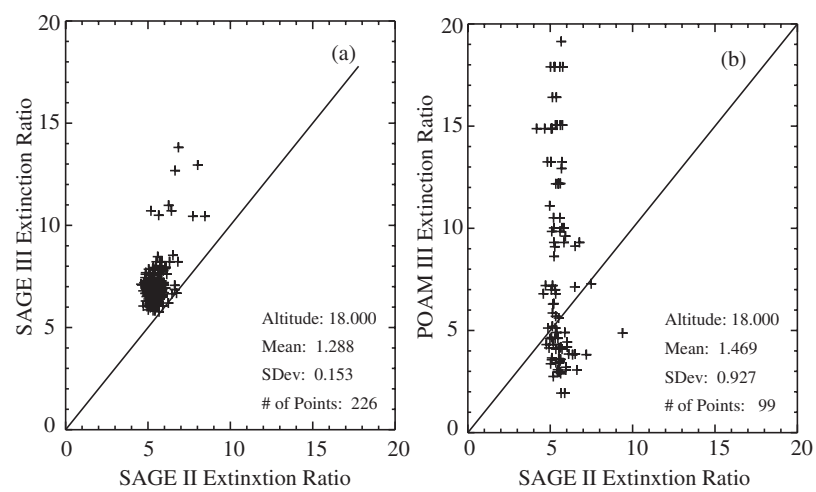

Fig. 13. This figure shows the relationship between (a) SAGE III and SAGE II aerosol extinction coefficient ratio pairs (449 to 1020$\mathrm{nm}$ and 452 to $1020-\mathrm{nm}$ ) and (b) POAM III and SAGE II aerosol extinction coefficient ratio pairs (442 to $1018-\mathrm{nm}$ and 452 to $1020 \mathrm{~nm}$ ) at $18 \mathrm{~km}$ matched using the criteria described in the text. The mean statistics shown were computed using the ratio defined in Eq. (3). The solid line has a slope of 1 .

errors in the extinction coefficient measurements as a function of wavelength are highly correlated for both instruments and do not fluctuate independently (Kent et al., 2006). The low dynamic range in the ratio (only about $\pm 15 \%$ ) leads to relatively low correlation coefficient values that lie near 0.3 over the entire depth of the profile. The POAM III to SAGE II comparisons can also be inferred from the results shown above. There is substantial structure in the altitude profiles of the individual comparisons shown in Figs. 9 and 12, which leads to the structure observed in Fig. 14. In this case, the ratio varies from 1.7 at $12 \mathrm{~km}$ to around 0.83 at $23 \mathrm{~km}$ and is generally near 1.5 between 15 and $20 \mathrm{~km}$. As shown above, the POAM III extinction at both $1 \mu \mathrm{m}$ and $450 \mathrm{~nm}$ is on average biased high compared to SAGE II, with a larger bias at $450 \mathrm{~nm}$. This leads to the generally positive bias in the wavelength ratio seen in Fig. 14. The large scatter in color ratio between these instruments is clearly seen in Fig. 13 where the range for POAM III ranges from values of about 2 to nearly 20 while SAGE II varies from 4 to 9 and SAGE III varies between 5.5 and 9 with a few points between 10 and 15. This results primarily from the large noise in the POAM III $1-\mu \mathrm{m}$ measurements, as described previously (Randall et al., 2001). Not surprisingly, the correlation between SAGE II and POAM III color ratios is for all intents and purposes zero. Since the SAGE II/SAGE III color ratios comparisons are well behaved and the POAM III/SAGE III problems are understood to be primarily the product of noise in the POAM III $1-\mu \mathrm{m}$ extinction coefficient data as shown in Fig. 1 and following figures, we conclude that the SAGE III color ratio data is reasonably reliable.

SAGE III and POAM III instruments show larger color ratios than the SAGE II instrument. This is consistent with comparisons (not shown) between the three instru-
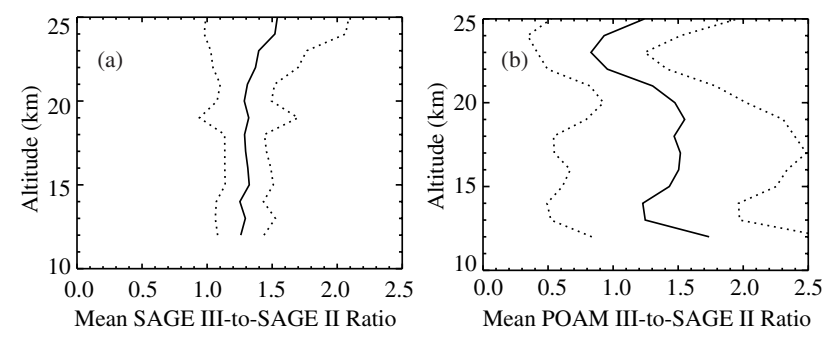

Fig. 14. (a) Mean profile of the ratio of SAGE III 449 to 1020-nm aerosol extinction ratio and the SAGE II $452 \mathrm{~nm}$ to $1020-\mathrm{nm}$ aerosol extinction ratio for the winters of 2003 through 2005. The solid line is the mean while the dotted lines are the 1-sigma confidence limits. (b) Same as frame (a) except for POAM III $442 \mathrm{~nm}$ to $1018 \mathrm{~nm}$ aerosol extinction ratio and the SAGE II 452 to 1020 -nm aerosol extinction ratio.

ments for more standard coincidence criteria (e.g., $\pm 24 \mathrm{~h}$, $500 \mathrm{~km}$ ). These comparisons comprise more than 2000 SAGE III/POAM III coincidences (many during the winter) and more than 300 SAGE III/SAGE II coincidences (none during the winter). These comparisons reveal that the color ratio for SAGE III is $20-30 \%$ higher than the color ratio for SAGE II, from $10-22 \mathrm{~km}$. This results from the fact that SAGE III extinction at $450 \mathrm{~nm}$ is $\sim 10-15 \%$ higher than SAGE II, whereas it is $10-15 \%$ lower at $1 \mu \mathrm{m}$. Given the very low background levels of aerosol extinction that persisted throughout the lifetime of SAGE III, this is quite reasonable agreement. As expected from the more variable POAM measurements at $1 \mu \mathrm{m}$, the color ratio comparison for SAGE III vs. POAM III shows more structure, but on average is within $\pm 20 \%$ from $\sim 13-21 \mathrm{~km}$. In this altitude range SAGE III and POAM III extinctions at $450 \mathrm{~nm}$ are within $\pm 15 \%$, while at $1 \mu \mathrm{m}$ POAM III is higher than SAGE III by 20-30\% (increasing to $40-60 \%$ below $15 \mathrm{~km}$ ).

We now return to the original question raised by the results of Russell et al. (2004) and shown in Fig. 1: are the differences between POAM III and SAGE III aerosol extinction at $1 \mu \mathrm{m}$ that are most obvious in the 2002-2003 winter indicative of a problem in the SAGE III data? We conclude that based on comparisons of both the SAGE III and POAM III data sets to a common standard, SAGE II, we have no evidence to suggest that there is any more error in the SAGE III data than in the other data sets. The precision of the SAGE III $1-\mu \mathrm{m}$ data is significantly higher than that of POAM III, which lends credibility to the SAGE III retrievals. On average, as seen from Fig. 14, the color ratio of SAGE III is intermediate between that of SAGE II and POAM III, another point that suggests the SAGE III measurements are not significantly in error. In addition, the lack of an appreciable altitude dependence in the relationship between the 1020 and 450-nm measurements by SAGE III and SAGE II (Figs. 9a and $12 \mathrm{a}$ ) suggests that these measurements are robust since most known sources of bias in these measurements are both 
strongly wavelength and altitude dependent (Thomason et al., 2007) ${ }^{1}$. Conversely, SAGE III extinctions at $1 \mu \mathrm{m}$ are lower than both SAGE II and POAM III, so this could point to a low bias in the SAGE III 1- $\mu$ m extinction measurements that might be at least partly responsible for the low values in winter. At this time, however, the causes of the overall biases between these three instruments (and the results of Russell et al., 2005) are not understood, and errors cannot be definitively attributed to one instrument instead of another. We also note that Alfred et al. (2006) concluded that the biases described by Russell et al. (2005) pertained to conditions of very low extinctions, and that the POAM III and SAGE III measurements of PSCs agreed well with each other.

\section{Summary and conclusions}

Based on this analysis, we find that the SAGE III data at both 1020 and $449 \mathrm{~nm}$ are reliable over a broad range of aerosol extinction values and suitable for use in PSC studies. Both of these channels show systematic bias relative to the SAGE II values that are nearly constant from 12 to $25 \mathrm{~km}$, but in opposite directions. In addition, the color ratios for these instruments, while reflecting the individual biases, remains highly robust and are extremely consistent as a function of altitude. We have not attempted to diagnose the source of the differences between the two SAGE instruments. Differences between POAM III and SAGE II, particularly at 1020 $\mathrm{nm}$, vary significantly with altitude, but the overall magnitude of the differences is similar to that between SAGE III and SAGE II. POAM III measurements are significantly less noisy at $450 \mathrm{~nm}$ than at $1 \mu \mathrm{m}$, so the comparisons with SAGE II at the shorter wavelengths are better behaved. As has been previously reported, the POAM III aerosol extinction data at $1 \mu \mathrm{m}$ is significantly noisier than the data from either SAGE instrument. The noise may contribute to the disagreement between instruments reported here. The disagreements reported by Russell et al. (2005) might arise in part from the small systematic low bias, currently not understood, that is observed between SAGE III and both SAGE II and POAM at $1-\mu \mathrm{m}$. In any case, we conclude that based on the above analysis that, beyond the modest biases reported here, there is no reason to believe that the SAGE III data is pathologically biased low within the polar vortex or that the low extinctions recorded by the instrument are anything but the product of geophysical processes.

Acknowledgements. The authors would like to thank G. Manney of JPL for providing a wealth of auxiliary data for SAGE II and SAGE III including the potential vorticity data used in this paper.

\footnotetext{
${ }^{1}$ Thomason, L. W., Burton, S. P., Luo, B.-P., and Peter, T.: SAGE II Measurements of Stratospheric Aerosol Properties at NonVolcanic Levels, submitted to Atmospheric Chemistry and Physics, February 2007.
}

Edited by: R. MacKenzie

\section{References}

Alfred, J., Fromm, M., Bevilacqua, R., et al.: Observations and analysis of polar stratospheric clouds detected by POAM III and SAGE III during the SOLVE II/VINTERSOL campaign in the 2002/2003 Northern Hemisphere winter, Atmos. Chem. Phys. Discuss., 6, 11391-11 426, 2006.

Chu, W. P., McCormick, M. P., Lenoble, J., Brogniez, C., and Pruvost, P.: SAGE II inversion algorithm, J. Geophys. Res., 94, 8339-8351, 1989.

Curtius, J., Weigel, R., Vössing, H.-J., Wernli, H., Werner, A., Volk, C.-M., Konopka, P., Krebsbach, M., Schiller, C., Roiger, A., Schlager, H., Dreilling, V., and Borrmann, S.: Observations of meteoric material and implications for aerosol nucleation in the winter Arctic lower stratosphere derived from in situ particle measurements, Atmos. Chem. Phys., 5, 3053-3069, 2005, http://www.atmos-chem-phys.net/5/3053/2005/.

Fromm, M., Alfred, J., and Pitts, M.: A unified, long-term, highlatitude stratospheric aerosol and cloud database using SAM II, SAGE II, and POAM II/III data: algorithm description, database definition, and climatology, J. Geophys. Res., 108, 4366, doi: 10.1029/2002JD002772, 2003.

Kent, G. S., Trepte, C. R., Farrukh, U. O., and McCormick, M. P.: Variation in the stratospheric aerosol associated with the north cyclonic polar vortex as measured by the SAM II satellite sensor, J. Atmos. Sci., 42, 1536-1551, 1985.

Lumpe, J. D., Bevilacqua, R. M., Hoppel, K. W., and Randall, C. E.: POAM III retrieval algorithm and error analysis, J. Geophys. Res., 107(D21), 4575, doi:10.1029/2002JD002137, 2002.

Manney, G. L., Michelsen, H. A., Bevilacqua, R. M., Gunson, M. R., Irion, F. W., Livesey, N. J., Oberheide, J., Riese, M., Russell III, J. M., Toon, G. C., and Zawodny, J. M.: Comparison of satellite ozone observations in coincident air masses in early November 1994, J. Geophys. Res., 106, 9923-9943, 2001.

McCormick, M. P., Chu, W. P., Grams, G. W., Hamill, P., Herman, B. M., McMaster, L. R., Pepin, T. J., Russell, P. B., Steele, H. M., and Swissler, T. J.: High-latitude stratospheric aerosols measured by the SAM II satellite system in 1978 and 1979, Science, 214, 328-331, 1981.

Poole, L. R. and Pitts, M. C.: Polar stratospheric cloud climatology based on Stratospheric Aerosol Measurement II observations from 1978 to 1989, J. Geophys. Res., 99, 13 083-13 089, 1994.

Poole, L. R., Trepte, C. R., Harvey, V. L., Toon, G. C., and VanValkenberg, R. L.: SAGE III observations of Arctic polar stratospheric clouds - December 2002, Geophys. Res., Lett., 30(23), 2216, doi10.1029/2003GL018496, 2003.

Randall, C. E., Bevilacqua, R. M., Lumpe, J. D., and Hoppel, K. W.: Validation of POAM III aerosols: Comparison to SAGE II and HALOE, J. Geophys. Res., 106(D21), 27 525-27 536, 2001.

Russell, P., Livingston, J., Schmid, B., Eilers, J., Kolyer, R., Redemann, J., Ramirez, S., Yee, J.-H., Swartz, W., Shetter, R., Trepte, C., Risley Jr., A., Wenny, B., Zawodny, J., Chu, W., Pitts, M., Lumpe, J., Fromm, M., Randall, C., Hoppel, K., and Bevilacqua, R.: Aerosol optical depth measurements by airborne sun photometer in SOLVE II: Comparisons to SAGE III, POAM III and airborne spectrometer measurements, Atmos. Chem. Phys., 
5, 1311-1339, 2005,

http://www.atmos-chem-phys.net/5/1311/2005/.

SAGE III Algorithm Theoretical Basis Document: Solar and Lunar Algorithm, LaRC 475-00-108, Version 2.1, 26 March 2002.

Strawa, A. W., Drdla, K., Fromm, M., Pueschel, R. F., Hoppel, K. W., Browell, E. V., Hamill, P., and Dempsey, D. P.: Discriminating Types Ia and Ib polar stratospheric clouds in POAM satellite data, J. Geophys. Res., 107(D20), 8291, doi:10.1029/2001JD000458, 2002.

Thomason, L. W. and Poole, L. R.: Use of stratospheric aerosol properties as diagnostics of Antarctic vortex processes, J. Geophys. Res., 98, 23 003-23 012, 1993.
Thomason, L. W., Zawodny, J. M., Burton, S. P., and Iyer, N.: The SAGE II Algorithm: Version 6.0 and On-going Developments, 8th Scientific Assembly of International Association of Meteorology and Atmospheric Sciences, Innsbruck Austria, July 10 $18,2001$.

Thomason, L. W. and Taha, G.: SAGE III Aerosol Extinction Measurements: Initial Results, Geophys. Res. Lett., 30, 33-1-33-4, doi:10.1029/2003GL017317, 2003. 